\title{
MIGRACIONES Y DIVERSIDAD FUNCIONAL. LA REALIDAD INVISIBLE DE LAS MUJERES
} MIGRATIONS AND FUNCTIONAL DIVERSITY. INVISIBLE REALITY OF WOMEN

\section{ROSA MARÍA DÍAZ JIMÉNEZ* YOLANDA MARÍA DE LA FUENTE ROBLES** ROCÍO MUÑOZ MORENO***}

\section{RESUMEN}

Ante la invisibilidad de la diversidad funcional en los estudios migratorios y el tímido interés de las migraciones en los estudios sobre discapacidad, este trabajo tiene como objeto hacer visible la diversidad funcional, especialmente a las mujeres con diversidad funcional en los procesos migratorios. A partir del análisis del material bibliográfico se explora la producción científica sobre las mujeres con discapacidad migrantes mediante una revisión sistematizada del fenómeno. Se perfila cierta tipología de personas que interactúan con la discapacidad y la migración: personas con discapacidad que realizan la migración; personas adultas que han accedido a la situación de discapacidad después del proceso migratorio o asociado al mismo y menores que acompañados por sus familias migran para buscar mejores sistemas de protección social que apoyen su discapacidad.

PALABRAS CLAVE: Mujeres con discapacidad, migraciones, género, personas refugiadas con discapacidad.

\section{ABSTRACT}

Given the invisibility of functional diversity in migratory studies and the timid interest of migrations in studies on disability, this work aims to make functional diversity visible, especially to women with functional diversity in migration processes. Based on the analysis of the bibliographic material, the scientific production on migrant women with disabilities is explored through a systematic review of the phenomenon.

A typology of people who interact with disability and migration is identified: persons with disabilities who perform the migration; adults who have accessed the disability situation after the migration process or associated with it and minors who, accompanied by their families, migrate to seek better social protection systems that support their disability.

KEYWORDS: women with disabilities, migrations, gender, refugees with disabilities.

\footnotetext{
* Doctora en Trabajo Social por la Universidad Pablo de Olavide. Profesora titular del Departamento de Trabajo Social y Servicios Sociales, Universidad Pablo de Olavide. Correo electrónico: rdiajim@upo.es

** Doctora en Derecho por la Universidad de Jaén. Catedrática de la Facultad de Trabajo Social de la Universidad de Jaén. Correo electrónico: ymfuente@ujaen.es

*** Doctora en Sociología por la Universidad Pablo de Olavide. Facultad de Ciencias Sociales, Departamento de Sociología. Universidad Pablo de Olavide, Sevilla. Correo electrónico: rmunmor@upo.es
} 


\section{INTRODUCCIÓN Y REVISIÓN SIS- TEMATIZADA COMO MÉTODO DE APROXIMACIÓN}

En los últimos años el fenómeno de las migraciones ha aumentado el interés científico a tenor del incremento de las investigaciones al respecto. Incluso el avance en estudios migratorios con perspectiva de género es notable. Sin embargo, la situación de personas con discapacidad en los procesos migratorios no ha sido un objeto analítico de interés equiparable, menos aún si enfocamos la situación específica de las mujeres.

La Organización Internacional de las migraciones reconoce la discapacidad como un elemento de vulnerabilidad de las personas migrantes. Las "personas migrantes vulnerables" son aquellas que aun sin reunir

las condiciones para recibir protección en virtud de los marcos para refugiados, en sus países de origen se enfrentan a una diversidad de situaciones que ponen en peligro su vida... o son objeto de discriminación por cualquier motivo (McAuliffe y Ruhs, 2018, p.159).

Este artículo se centra, en primer lugar, en analizar los datos y las tendencias mundiales fundamentales sobre migración y en ningún caso aparece reflejada la situación de personas con discapacidad migrantes. La utilización de diferentes conceptos, definiciones, y métodos de recopilación de datos, tanto de migración como de discapacidad obstaculizan la comparabilidad de las estadísticas.

De las escasas estimaciones poblacionales de las personas con diversidad funcional migrantes, observamos una gran dificultad para realizar una aproximación a las cifras que sea válida a nivel internacional. Del estudio sobre las personas inmigrantes con discapacidad en España de Díaz y Huete, pionero en este ámbito, destaca que las personas inmigrantes con discapacidad suponen el $5 \%$ de la población inmigrante española. De esta población, el 53,8\% son mujeres (Diáz y Huete, 2008, p. $50)$.

Para hacer visible la realidad de esas mujeres, se ha realizado un análisis de fuentes secundarias de carácter bibliográfico. El objetivo del trabajo ha sido explorar la producción científica sobre las mujeres con discapacidad migrantes mediante una revisión sistematizada del fenómeno siguiendo a Codina, ya que aplicamos algunos criterios de sistematización de la información (Codina, 2018, p. 10). En la fase de búsqueda se tienen en cuenta base de datos como WOS y SCOPUS, y como criterios de inclusión se han tenido en cuenta la discapacidad y las migraciones, la discapacidad y personas refugiadas, la discapacidad y la inmigración, el género las migraciones y la 
discapacidad, así como específicamente a las mujeres con discapacidad migrantes. Se han excluido las aportaciones no relacionadas con las ciencias sociales. Dada la escasez de trabajos de las bases de datos mencionadas se han incorporado otras aportaciones en castellano de las bases de datos DIALNET y REDALYC. En estas dos últimas bases de datos solamente hemos encontrado dos artículos en cuyo título se trate la cuestión de las mujeres con discapacidad migrantes o inmigrantes. social (Montesino-Parra, 2016). En el caso de los trabajos de la sociología de la educación es muy frecuente analizar la inmigración en la educación especial, o bien en el contexto de los factores de riesgo de la juventud inmigrante. (Portera, 2014)

A partir del análisis del material bibliográfico se presentan los resultados en tres partes, un primer apartado que relaciona la discapacidad y las migraciones, un segundo que se refiere a los hallazgos sobre las personas con

TABLA 1. HALLAZGOS EN BASES DE DATOS SOBRE MUJERES DISCAPACIDAD Y MIGRACIONES

\begin{tabular}{|l|c|c|}
\multicolumn{1}{|c|}{ CRITERIOS DE INCLUSIÓN } & WOS & SCOPUS \\
\hline MIGRATIONS AND DISABILITY (Título) & 31 & 18 \\
\hline REFUGEE AND DISABILITY (Título) & 45 & 43 \\
\hline $\begin{array}{l}\text { GENDER AND MIGRATIONS(Título+abstract) } \\
\text { AND DISABILITY (Título) }\end{array}$ & 18 & 15 \\
\hline $\begin{array}{l}\text { WOMAN AND MIGRATIONS(Título+abstract) } \\
\text { AND DISABILITY (Título) }\end{array}$ & 10 & 11 \\
\hline Total & 104 & 87 \\
\hline
\end{tabular}

Fuente. Elaboración propia a partir de la búsqueda de información en base de datos WOS y SCOPUS diciembre 2018.

Aunque se observa un incremento de producción sobre mujeres migrantes, cuestiones relativas a mujeres con discapacidad migrantes se tratan de manera escasa o tangencial. Otra de las cuestiones que aporta dificultades de identificación de trabajos es la tendencia observada a considerar o equiparar la situación de muchas personas migrantes a la discapacidad. Incluso se analiza como discapacidad discapacidad refugiadas y uno más ya centrado en la discusión de los estudios específicos vinculados con las mujeres con diversidad funcional migrantes.

\section{MIGRACIONES Y DIVERSIDAD FUN- CIONAL}

Cada año, existen millones de personas que se 
ven forzadas a abandonar su país de origen, siendo obligadas a desplazarse como consecuencia de diferentes desastres (tanto naturales como propiciados por el ser humano). Según datos de ACNUR, en el año 2017, 68,5 millones de personas se han visto forzadas a huir. Todo ello, en una tendencia de aumento en el número de desplazamientos que se ha producido en los últimos cinco años (ACNUR, 2017)

Paralelamente, con relación al tema de la ampliación de derechos en el ámbito de la diversidad, en el panorama internacional ha ido creciendo la importancia y la necesidad de reconocer los derechos de las personas con diversidad funcional. Prueba de ello es la entrada en vigor de la Convención de Naciones Unidas sobre los Derechos de las personas con Discapacidad, que ha generado un interés sin precedentes en los derechos de este colectivo. La rápida ratificación de este documento por los diferentes estados muestra la importancia concedida a este instrumento, sólo comparable a la aceptación obtenida por la Convención de los derechos del Niño. Este documento define a las personas con discapacidades como: "Aquellos que tienen impedimentos físicos, mentales, intelectuales o sensoriales a largo plazo, que en interacción con varias barreras pueden impedir la plena $y$ efectiva participación en sociedad en igualdad de condiciones con los demás." (art.1)

La Organización Mundial de la Salud (OMS) es- tima que alrededor del 15,6\% de la población mundial tiene alguna clase de discapacidad. La estimación es considerablemente más alta entre los países de menos ingresos, así como entre las zonas más pobres de cada comunidad.

La creciente importancia de estas dos cuestiones sociales en el ámbito internacional lleva a preguntarse por las interrelaciones que se producen entre ambas. Si la población con discapacidad y la población migrante por separado son objeto de actuación política por la vulnerabilidad de su situación, el hecho de ser migrante y tener alguna discapacidad merece ser enfocado dada la interacción de desigualdades a las que se enfrenta el colectivo.

Por ello, con la finalidad de diseñar una adecuada planificación que permita el desarrollo de una actuación política eficaz, se reclama la necesidad de estudiar las características que presenta la población migrante con discapacidad, así como las dificultades específicas a las que se enfrenta en los diferentes contextos. Dentro de este colectivo, se distinguen tres perfiles mayoritarios: 1) personas que emigran y posteriormente desarrollan una discapacidad en el país de destino; 2) personas que emigran y en el transcurso de ese desplazamiento se desarrolla o genera algún tipo de discapacidad (desplazamientos forzosos, refugiados o estancias en campos de concentración); y 3) familias que emigran precisamente por tener entre sus miembros alguna persona con 
discapacidad, con la intención de mejorar el acceso a los servicios y a las políticas sociales, o debido a políticas restrictivas en el país de origen en cuanto al empleo o la aceptación de las personas con algún tipo de discapacidad (Díaz, Huete y Jiménez, 2009)

En el marco de la revisión de la literatura al respecto, la mayor parte de las investigaciones que se han llevado a cabo coinciden en la necesidad de profundizar en los estudios que analicen estos fenómenos de manera conjunta dada la escasa literatura existente, así como a la falta de estandarización de las herramientas que tratan de medir la discapacidad entre la población migrante, derivada de la ausencia de una definición común que se aplique en el conjunto de los países.

Autores como Smith-khan et al. (2014) han centrado su estudio en el análisis de las herramientas utilizadas por los distintos organismos internacionales para la identificación de la discapacidad entre la población migrante. Una de las mayores dificultades que encuentran es la falta de consenso en torno al modo en el que se define y se operativiza el término discapacidad en los diferentes contextos y herramientas para la recogida de información. En este sentido, se considera necesario el establecimiento de estándares comunes, que vayan desde una definición compartida del concepto hasta una estandarización y sistematización de los métodos diseñados para la recogida de información, que permitan la correcta identificación y el acceso a la información y comparabilidad de los datos a nivel internacional.

Asimismo, estos autores señalan que la identificación de la discapacidad también se encuentra supeditada al grado en el que la persona percibe que será tratada en el país de destino debido a su estatus de migrante con discapacidad.

En este sentido, en la medida en la que se perciba que los estados receptores excluyen a estas personas o prefieren a aquellos con buen estado de salud, tienden a no identificarse como tal, evitando así el estigma social del que perciben que podrán ser objeto.

En esta línea, autores como Hon-Sing Wong (2012) y Montesino-Parra (2016) nos ayudan a entender el modo en el que políticas migratorias con un elevado enfoque "capacitista", como es el caso de Suecia o Canadá, contribuyen a "patologizar" a este colectivo en base a la propia concepción de la discapacidad. La mala imagen que históricamente se le ha dado a la movilidad en Suecia, unido a la conceptualización de la condición de refugiado como "discapacidad social" ha sido uno de los mayores obstáculos para la integración de refugiados en Suecia.

Además de la estandarización y sistematización de las herramientas para la identificación y la recogida de información sobre las necesidades y las características de la población migrante con discapacidad, se considera ne- 
cesario aumentar la formación al personal de las organizaciones destinado a atender a este colectivo con la finalidad de identificar de un modo más sensible las diferentes maneras en las que puede manifestarse la discapacidad (Smith-khan et al, 2014)

En la relación que se produce entre la discapacidad y la migración intervienen otros muchos factores, como puede ser el género, la edad, la clase social, el tipo de discapacidad, el tipo de políticas en el país de origen y de destino y el acceso a las mismas.

Resultados obtenidos en estudios, como el de Graefe et al (2006) muestran que, aunque en términos generales una familia integrada por algún miembro con discapacidad sea menos propensa a emigrar, si encuentra dificultades para acceder al mercado laboral, así como a las políticas de bienestar en su país de origen tendrá mayores probabilidades de iniciar el proyecto migratorio en búsqueda de mejores condiciones.

Findley (1988) analiza la direccionalidad de la relación salud-migración en Estados Unidos mediada por algunos factores como el género o la edad. Algunos de los resultados encontrados en relación al género muestran que las mujeres experimentan un mayor deterioro de la salud tras la migración y asimismo presentan una mayor probabilidad de migrar tras experimentar algún problema de salud. En atención a la edad, el estado de salud se transforma en un impedimento para emigrar en el caso de la población de mayor edad, mientras que para la población joven que padece algún tipo de enfermedad crónica no sólo no supone un impedimento, sino que presentan una mayor probabilidad de migrar. Asimismo, la población que emigra a mayor edad tiene más posibilidades de sufrir un deterioro de salud en el periodo post-migratorio, que de iniciar el proceso migratorio tras un deterioro de salud. Por otra parte, la población que ha migrado con alguna discapacidad tiene mayores probabilidades de desestabilizarse tras el proceso migratorio.

Otros trabajos, como el de García y Reyes (2018) en su análisis de las trayectorias de la población mayor mexicana residente en EE.UU., encuentran relación entre salud y migración en función de la edad a la que se inicie el proyecto migratorio. De esta forma, encuentran que la cohorte de población más afectada por el proceso migratorio en términos de salud es la que inicia el proyecto migratorio a mediana edad, mientras que la población más joven se asimila a los datos presentados por la población nativa o incluso presentan unos resultados algo superiores en términos de salud. En el caso de la población que migra a edad tardía, a pesar de que puedan presentar menores oportunidades para integrarse social y económicamente, cuentan con mayor probabilidad de conservar factores sociales y culturales de protección de la salud, por lo que se ven menos afectados por este proceso. 
El hecho de que sea la población de mediana edad la que presenta mayor deterioro de la salud como consecuencia de este proceso, a pesar de contar de partida con una buena salud que le permite incorporarse al mercado laboral de manera efectiva, puede tener que ver con las condiciones de trabajo a las que se ha visto expuesta esta cohorte de población, con escaso acceso a políticas sociales y unas condiciones de vivienda infradotadas ${ }^{1}$.

En esta era de "superdiversidad" (Vertovec, 2007, en Burns, 2017) los derechos de los individuos son un tema clave. En el ámbito de los derechos humanos, Naciones Unidas ha desarrollado diferentes herramientas que tratan de ofrecer un marco para la protección de las personas desplazadas - Convención para los Derechos de los Refugiados (Naciones Unidas, 1951) y la Convención para la Protección de los derechos de todos los Trabajadores Migrantes (Naciones Unidas, 1990)- así como una serie de convenciones que existen para asegurar estos derechos, incluyendo a personas con discapacidad, como la Convención de Naciones Unidas sobre los Derechos de las Personas con Discapacidad, que establece:

\section{Bracero Programm (1942-1964)}

2 "Superdiversidad" Es definida por la complejidad, "se distingue por la interrelación económica de variables entre un número cada vez mayor de nuevos perfiles de inmigrantes, en pequeños grupos, dispersos y procedentes de múltiples orígenes, conectados trasnacionalmente, socioeconómicamente diferenciados y legalmente estratificados" (Vertovec 2007, p.1024)
Libertad de desplazamiento y nacionalidad - Las personas con discapacidad tienen derecho, en igualdad de condiciones con las demás, a adquirir una nacionalidad, atravesar fronteras y cambiar de nacionalidad. Los niños con discapacidad serán inscritos inmediatamente después de su nacimiento y tendrán desde el nacimiento derecho a un nombre, a adquirir una nacionalidad y, en la medida de lo posible, a conocer a sus padres y ser atendidos por ellos. (Organización de Naciones Unidas, 2006, art. 18)

No obstante, el acceso a estos derechos por parte de la población migrante se encuentra supeditado a la obtención de la ciudadanía, por lo que el acceso a los recursos depende de la adaptación que los diversos estados aplican de los diferentes documentos internacionales. Todo ello encuadrado en el contexto derivado de la crisis financiera de 2008, unido al mayor peso que están adquiriendo los partidos de derecha en Europa, la inmigración se presenta a menudo en el discurso público como problema. Los estados perciben a la población migrante, especialmente aquella con algún tipo de discapacidad como una carga o un gasto excesivo para las arcas del estado. (Burns, 2017).

A la luz de la revisión realizada sobre la materia, se expone la necesidad de seguir ahondando en las interrelaciones que se producen entre 
migración y discapacidad al objeto de construir un marco teórico que permita comprender de manera profunda la multidimensionalidad de factores que pueden afectar a esta relación.

Asimismo, se reclama la importancia de recoger información en diferentes contextos territoriales y con distintos perfiles de población, dada las diferencias culturales y normativas específicas de cada territorio. En esta línea, la estandarización y sistematización de las herramientas utilizadas para identificar la discapacidad de la población migrante, así como para la recogida de información que permita su análisis y comparabilidad, debe ser un aspecto prioritario de la agenda política de los diferentes países, por lo que deben desarrollarse los mecanismos necesarios que garanticen su efectivo cumplimiento. Sin un adecuado diagnóstico de la realidad y una voluntad política real de intervenir y garantizar la plena inclusión todas las personas, el esfuerzo por la planificación y el diseño de políticas públicas efectivas parece destinado al fracaso. en la estrategia europea que creara la red necesaria para definir nuevas políticas sociales que dieran solución a una ingente lista de necesidades que venían migrando sin respuesta.

En 2014 el número de personas que habían solicitado por primera vez ser reconocidas como refugiadas fue de 562.675 (mujeres: 164.155), en 2015 esta cifra aumentó considerablemente hasta alcanzar 1,257.035 (mujeres: 344.390), en 2016 descendió ligeramente a 1.206.115 (mujeres: 389.165), cayendo en 2017 a una cifra similar a la de 2014 a 619.685 (mujeres: 204.355) ${ }^{3}$ como consecuencia de las políticas de contención acordadas. De todas ellas, las personas refugiadas, las solicitantes de asilo y otras migrantes con discapacidad, no son identificadas adecuadamente y no cuentan con un acceso igualitario a servicios en los centros de acogida.

El SECA (Sistema Europeo Común de Asilo) recoge el conjunto de normas a nivel de la Unión Europea desde 2003 que competen a personas refugiadas con discapacidad, entre ellas destacan:

- La Directiva 2003/9/CE del Consejo de 27 de enero de 2003 por la que se aprueban normas mínimas para la acogida de los solicitantes de asilo en los Estados miembros.

Entre 2015 y 2016 se produjo una entrada masiva de solicitantes de protección internacional a la Unión Europea; ese lapso temporal fue el punto de inflexión para iniciar un cambio
- La Directiva 2005/85/CE del Consejo de 1 de diciembre de 2005 sobre normas mínimas

3 http://appsso.eurostat.ec.europa.eu/nui/show.do?dataset=migr_asyappctzm 
para los procedimientos que deben aplicar los Estados miembros para conceder o retirar la condición de refugiado garantiza el acceso a un procedimiento de asilo justo y eficaz.

- La Directiva 2011/95/UE del Parlamento Europeo y del Consejo de 13 de diciembre de 2011 por la que se establecen normas relativas a los requisitos para el reconocimiento de nacionales de terceros países o apátridas como beneficiarios de protección internacional, a un estatuto uniforme para los refugiados o para las personas con derecho a protección subsidiaria y al contenido de la protección concedida determina que "Procede garantizar el acceso a la asistencia sanitaria, atención de la salud tanto física como psíquica, a los beneficiarios de protección internacional."

- La Directiva 2013/33/UE del Parlamento Europeo y del Consejo de 26 de junio de 2013 por la que se aprueban normas para la acogida de los solicitantes de protección internacional incluye en su artículo 21 a los refugiados con una discapacidad en el colectivo de las personas especialmente vulnerables:

Existen además normas internacionales que protegen en especial a las personas refugiadas con una discapacidad. Así por ejemplo el artículo 11 de la Convención sobre los Derechos de las Personas con Discapacidad de las Naciones Unidades. La Asamblea General de las Naciones Unidas aprobó el 19 de septiembre de 2016 la Declaración de Nueva York para los Refugiados y los Migrantes, donde se señala que en los Estados atenderán en este ámbito a las personas con discapacidad.

En concreto, se comprometen a utilizar el proceso de inscripción para planificar las medidas de apoyo personas refugiadas con necesidades especiales de protección entre quienes reconoce a las personas con discapacidad.

En respuesta a las recomendaciones del Comité, el Parlamento Europeo adoptó la Resolución del Parlamento Europeo, de 7 de julio de 2016, sobre la aplicación de la Convención de las Naciones Unidas sobre los Derechos de las Personas con Discapacidad, con especial atención a las Observaciones finales del Comité sobre los Derechos de las Personas con Discapacidad de las Naciones Unidas (2015/2258(INI)) Parlamento Europeo que en el apartado 58 reconoce la necesidad de garantizar la cobertura de derechos y servicios a personas con discapacidad redoblando esfuerzos en caso de personas refugiadas y solicitantes de asilo e insiste en la necesidad de integrar la discapacidad en las políticas de la Unión Europea en el ámbito de la migración y las personas refugiadas; en su apartado 59 pide a la Comisión y al Consejo, de conformidad con el artículo 11 de la CDPD, "que cuando elaboren propuestas para solucionar la cuestión de los refugiados, en lo relativo a la financiación o a otras medidas de apoyo, prevean atención especial para las personas con discapacidad" 
A todo ello hay que añadir, que la diversidad entre los diferentes Estados miembros no sólo es considerable en lo relativo a la cifra de solicitudes presentadas, sino también en cuanto a las políticas públicas, los discursos de los respectivos gobiernos y la respuesta de la sociedad civil. No obstante, el denominador común de todos los países son las deficiencias en los sistemas de acogida de este colectivo que presenta un grave riesgo de vulnerabilidad y exclusión. Son especialmente insuficientes los sistemas de atención a los/as solicitantes de protección internacional y los/as refugiados/as con una discapacidad y/o situación de dependencia, a menudo causado por situaciones traumáticas sufridas en los países de origen o durante la huida.

Entre los déficits identificados están los problemas en el diagnóstico de las necesidades específicas de las personas con discapacidad y/o en situación de dependencia, restricciones legales que impiden que éstos puedan acceder a los servicios regulares de atención, falta de accesibilidad de las instalaciones de acogida, falta de ofertas para la inserción laboral, insuficiente cooperación entre los sistemas encargados de la acogida de refugiados y aquellos que son competentes de la atención a personas con discapacidad.

La agencia para los refugiados de la ONU (ACNUR), organizaciones internacionales y locales de asistencia que trabajan con centros de refugiados en Grecia trasladaron a Human
Rights Watch ${ }^{4}$ que cuentan con muy pocos o ningún programa, diseñado específicamente para responder a los derechos y las necesidades de los solicitantes de asilo, refugiados y otros migrantes con discapacidad. En función de las investigaciones realizadas en Grecia entre 2016 y 2017, Human Rights Watch concluyó que en Grecia no se identifica adecuadamente a solicitantes de asilo y refugiados con discapacidad, en parte debido a que el proceso de registro es apresurado y a la necesidad de que el personal reciba mayor orientación. Sin una comprensión adecuada de la magnitud y las necesidades, los organismos de asistencia no pueden responder con eficacia.

Para poner fin a esta doble discriminación, la Unión Europea debería solicitar información a sus estados miembros sobre la ejecución de sus programas para garantizar que los proyectos que financian beneficien a las personas con discapacidad y a otros grupos en riesgo. Todo ello sucede a pesar de que las diversas Directivas Europeas y las normas internacionales son taxativas, instando a los Estados miembros a tener en cuenta estos y otros colectivos especialmente vulnerables, el cumplimiento de las mismas suele ser una excepción.

Para sentar las bases de una nueva manera de proceder para corregir desajustes en la inter-

\footnotetext{
4 https://www.hrw.org/es/news/2017/01/18/grecia-refugiados-con-discapacidad-son-ignorados-y-relegados
} 
vención, se celebró en Bruselas la conferencia sobre "La situación de los refugiados y los migrantes con discapacidad" (2017), al objeto de llamar la atención sobre este grupo especialmente vulnerable de refugiados e inmigrantes, tratando de concienciar sobre los derechos y necesidades de las personas con discapacidad a través de las organizaciones internacionales que trabajan con ellos; y lo más significativo es que se recordó que la normativa europea para la acogida de los solicitantes de asilo en Europa, exige de los distintos Estados miembros que tengan en consideración la situación específica de las personas vulnerables, especialmente con relación a las condiciones de recepción, valorando individualmente sus necesidades particulares, en concreto, aquellas relacionadas con alguna discapacidad. Uno de los problemas destacados es la falta de una respuesta homogénea por parte de los Estados miembros a la hora de ofrecer protección a las personas vulnerables que llegan a Europa en busca de asilo, por lo que, en muchos casos, la integración de los migrantes con discapacidad, así como el acceso a derechos sociales es todavía precario.

Entre las principales conclusiones del encuentro, es interesante destacar que: 1) debe aplicarse por completo el artículo 11 de la Convención de Derechos de las Personas con Discapacidad, que exige a los Estados participantes adoptar todas las medidas necesarias para asegurar la protección y la seguridad de las personas con discapacidad en situación de riesgo; es necesario tener datos precisos del número de personas con discapacidad entre los refugiados y migrantes, hasta la fecha, no se dispone de registro fiable; 2) hay que garantizar el acceso a las solicitudes de asilo adaptándose a las personas con discapacidad; 3) es preciso adoptar un enfoque global a todos los derechos básicos (atención médica, vivienda o educación entre otros), teniendo presente la diversidad funcional. 4) la cooperación entre las distintas organizaciones e instituciones que trabajan con refugiados y personas con diversidad funcional; 5) hay que avanzar en el sistema de reasentamiento y acortar los plazos para la reunificación familiar en casos de vulnerabilidad y 6 ) es preciso reforzar las capacidades de las autoridades locales (Cauces, 2017, pp. 67-68)

Como consecuencia de lo anterior, el conocimiento que se tiene sobre la incidencia y la casuística concreta de la discapacidad en el colectivo de los solicitantes de protección internacional es francamente pobre. La mayoría de los estudios toca diferentes formas de vulnerabilidad y analiza la situación campos de refugiados en países vecinos a los de origen (HelpAge y Handicap Internacional 2014). Los informes de la Women's Commission for Refugee Women (2008, 2013) describe la situación de personas refugiadas en campamentos localizados en zonas urbanas en cincos países en vías de desarrollo y de re- 
fugiados sirios en el Líbano. Robert (2000), Roberts y Harris (2002) y Ward et al. (2008) analizan la atención prestada a refugiados con una discapacidad en el Reino Unido; Mirza y Heinemann (2012) detallan la situación en EEUU.

Varios estudios realizados por Handicap International en Alemania detallan los retos a los que se enfrenta la atención a los refugiados, situación extrapolable al resto de los países de la UE: 1) déficit en el diagnóstico y la identificación de las necesidades de atención de los refugiados con una discapacidad, lo que lleva a una pérdida considerable de tiempo y el traslado de estos refugiados a centros de acogida que no disponen de los recursos para garantizar un tratamiento adecuado; 2) restricciones legales que impiden que los refugiados con una discapacidad puedan acceder a los servicios regulares de atención; 3) falta de accesibilidad de las instalaciones (centros de acogida, academias de idiomas, instituciones); 4) falta de recursos para el aprendizaje del idioma adecuados a las necesidades de las personas con una discapacidad; 5) falta de ofertas específicas para la inserción laboral de los refugiados con una discapacidad; 6) desconocimiento de las estructuras de apoyo para las personas con una discapacidad por parte de los propios refugiados; 7) cooperación insuficiente entre los sistemas encargados de la acogida de refugiados y de la atención a personas con discapacidad; 8) excesiva complejidad y burocratización de los sistemas de atención de las personas con una discapacidad; 9) las iniciativas de la sociedad civil para el apoyo de los refugiados no dan abasto ante la complejidad que supone el apoyo de refugiados con una discapacidad y 10) falta de pisos adaptados que permitan la integración de los refugiados con una discapacidad (Schwalgin, 2017).

La Comisión Europea ha identificado doce retos principales entre los que figura la inmigración, como realidad que han de afrontar prácticamente todas las sociedades europeas (Comisión Europea 2016b y 2016c).

Así, por ejemplo, las políticas de integración de personas inmigrantes pertenecen al campo de actuación local debido a varios motivos: los municipios son el nivel administrativo adecuado para implementar políticas de cercanía, la lógica de las políticas municipales es diferente a la de los estados centrales, y solamente la política municipal puede movilizar los recursos locales, tanto los formales como los informales (Gebhardt, 2015).

En materia de inmigración e integración la perspectiva de los municipios es por lo tanto radicalmente diferente a la de los gobiernos centrales (Scholten y Penninx, 2016; Ambrosini, 2013, p. 137). De hecho, en este conjunto de políticas, los municipios han tenido un papel pionero, impulsando políticas de integración, obviando las críticas del gobierno central, dado que no pueden cerrar los ojos ante pro- 
blemas acuciantes e inmediatos relacionados con la inmigración (Zelani et al., 2016). En algunos países, como los Países Bajos (Leerkens, 2016) o Alemania (Aumüller et al., 2016: 30 f.) los gobiernos centrales han acabado asumiendo postulados e instrumentos de las políticas municipales.

\section{MUJERES CON DIVERSIDAD FUN- CIONAL MIGRANTES.}

Como puede apreciarse de lo mencionado anteriormente, las personas migrantes con diversidad funcional están sujetas a situaciones de “multi-exclusión" por la confluencia e interacción de diversos factores de vulnerabilidad que coinciden con la escasez de documentación administrativa, precariedad en el acceso a bienes y recursos, materiales culturales y sociales o el desarraigo y escasez de redes (Peñaherrera y Cobos, 2009, p. 43), situaciones que se agudizan en el caso de las mujeres aunque haya pocas evidencias.

Díaz y Huete plantean que hay dos factores fundamentales que influyen con respecto al género: la discriminación por cuestión de género en la cultura de procedencia y la discriminación por cuestión de género de la propia sociedad de destino y aun en estas circunstancias, las mujeres con discapacidad inmigrantes con entornos favorables tienen más éxito en los procesos de integración que los varones, mostrando más capacidad de adaptación (Díaz y
Huete, 2008, p. 193)

La relación entre mujeres migrantes y la discapacidad parece incrementar la resiliencia frente a las adversidades. Otros estudios muestran cómo las mujeres inmigrantes con discapacidad generan más capacidad para incluirse en la cultura del país de destino y generar redes para así conseguir el acceso a los sistemas de protección para sus hijos con discapacidad (Sajquim de Torres, M. y Lusk, M., 2018; Hyangsook, 2017).

Otras autoras como Dossa, muestran cómo la discapacidad aumenta la capacidad de lucha por el acceso a los derechos de la sociedad de destino y la generación de herramientas de activismo político. (Dossa, 2009) Sin embargo y aunque de forma no concluyente, otros toman la migración materna como factor de riesgo de discapacidad del desarrollo de sus hijos (Abdullahi, Wong, Mutch, et ál., 2019)

La mencionada "multiexclusión" se aborda en gran parte de los trabajos analizados desde la necesidad de la perspectiva de la interseccionalidad, enfocando discapacidad, migración, clase social y género (Dossa, 2009; Oliver y Singal, 2017)

Desde la antropología, la interseccionalidad y el feminismo antirracista, la doctora Parin Dossa muestra narraciones compartidas de mujeres inmigrantes musulmanas con discapacidad y una madre de dos hijos con discapacidad, instaladas en Canadá. Dossa da voz a las mujeres que muestran la dureza de la 
lucha por mantener la dignidad en la sociedad canadiense y ante sus iguales de origen, para entender las desigualdades que surgen cuando interactúan aspectos raciales, el género y la discapacidad (Dossa, 2005). Desde una visión crítica explica cómo se insertan en comunidades donde se sigue conceptualizando la discapacidad desde formas dominantes, por lo que cobra sentido entenderla desde la desigualdad en el acceso a recursos en los sistemas de protección y la opresión. Dossa reivindica un espacio de la discapacidad en el feminismo, espacio que aún no tiene (Dossa, 2009)

Algunos estudios del ámbito demográfico y de la salud documentan sistemáticamente una ventaja de salud de personas inmigrantes hispanas frente a las nativas cuando comparan la prevalencia de discapacidad, situación que revierte en la vejez, fundamentalmente por trayectorias laborales de baja cualificación (Sheftel, 2017; Garcia, Downer, Crowe, y Markides, 2017; Sheftel y Heiland, 2018; Garcia, Reyes y Rote, 2019). Las mujeres migrantes son las más saludables y la discapacidad es una consecuencia de una situación discriminatoria en el empleo, y es discriminatoria porque en el caso de los migrantes varones no se da ese retroceso en la salud.

Desde el ámbito de la promoción de la salud y mediante estrategias cualitativas, las autoras muestran cómo la salud de madres inmigrantes de niños y niñas con discapacidad está in- fluenciada por la interseccionalidad de factores sociales, incluyendo el estado migratorio, el género y la discapacidad (Khanlou et al., 2017)

Juana Morcillo relacionaba a mujeres con discapacidad intelectual y migrantes mediante la violencia de género (Morcillo, 2012) Otros estudios las relacionan comparando la salud mental de ambas en período perinatal Ayre K, Khalifeh H (2017). En este caso lo que las une son las situaciones de vulnerabilidad y pobreza.

Desde la sociología de la educación, y entorno disciplinar educativo, diversos estudios se centran en familias de migrantes con hijos con discapacidad (Oliver y Singal, 2017; Stevens, 2010; Gabel, Curcic, Powell, Khader, and Albee, 2009) alertando algunos de ellos sobre la escasa atención prestada en los estudios sobre alumnado migrante y los procesos de inclusión educativa a las necesidades educativas especiales y la diversidad funcional (Calatayud, González y Jiménez, 2017)

Oliver y Singal acuden a la interseccionalidad desde el punto de vista analítico. En un trabajo basado en un estudio de caso de una escuela especial en Inglaterra, explora cómo las interacciones de las familias migrantes con las escuelas se moldean tanto por sus itinerarios migratorios como por los procesos de inclusión y resalta la importancia de los apoyos lingüísticos y sociales en la tarea de la escuela. El enfoque interseccional le resulta útil 
para interpretar las interacciones familias y profesionales, sus encuentros, tensiones y dilemas que surgen de las intersecciones específicas de discapacidad, migración, clase social y género (Oliver y Singal, 2017)

Otros estudios explican la desproporcionalidad de la migración y grupos étnicos en la educación especial (Ferri y Connor, 2005) teniendo análisis comparativos de casos en diversos contextos internacionales. Se entiende la desproporcionalidad como la sobre o la sub-representación de grupos de estudiantes (migrantes y grupos étnicos) en las escuelas. Los autores muestran el alto porcentaje de estudiantes no nativos clasificados como estudiantes con discapacidad de aprendizaje y por tanto sujetos a programas de educación especial (Gabel, Curcic, Powell, Khader, and Albee, 2009) Junto con las personas con discapacidad en general, los grupos étnicos que tienen tasas más altas de clasificación en educación especial tienen resultados educativos deficientes y están en desventaja económica.

En el caso de los estudiantes inmigrantes e indígenas, la educación especial se emplea como una herramienta para la asimilación en la sociedad dominante, pero funciona como una herramienta de exclusión. El resultado es la alienación de los niños inmigrantes y una barrera adicional que enfrentan los niños indígenas y con diversidad funcional cuando intentan participar plenamente (Wehmeyer y Schwartz, 2001) Incluso en las minorías étnicas de Europa, que gozan de una buena situación económica, las minorías étnicas en general tienen bajos niveles de educación, dificultades de idioma, altas tasas de deserción escolar y tasas más altas de desempleo o subempleo. La desproporcionalidad en la educación especial como fenómeno global está intrínsecamente relacionada con cuestiones sociales más amplias, como la situación y desventaja socioeconómica, la integración en el mercado laboral y la inclusión social.

\section{CONCLUSIONES}

Tras el análisis y la discusión se pone de manifiesto la escasa producción científica, así como la falta de estandarización de las herramientas con perspectiva de género que midan la diversidad funcional entre la población migrante, todo ello derivado de la ausencia de una concepción común sobre el fenómeno.

Puede hablarse de invisibilidad de la diversidad funcional en los estudios migratorios y un tímido interés de las migraciones en los estudios sobre discapacidad. La falta de datos sobre personas migrantes con diversidad y a su vez desagregados por género provoca que estemos frente a un sector de población oculto y sin una comprensión adecuada de la magnitud y las necesidades las instituciones públicas o privadas que deben garantizar sus derechos no resultan eficaces. De hecho, aunque las normas nacionales e internacionales 
son taxativas a la hora de proteger este tipo de situaciones especialmente vulnerables, el cumplimiento de estas suele ser una excepción.

Ciertas políticas migratorias con un elevado enfoque "capacitista", contribuyen a "patologizar" a este colectivo, una de cuyas consecuencias es la ocultación. La identificación de la discapacidad en este caso se encuentra supeditada al grado en el que la persona percibe que será tratada en el país de destino debido a su estatus de migrante con discapacidad. En este sentido, en la medida en la que se perciba que los estados receptores excluyen a estas personas o prefieren a aquellos con buen estado de salud, tienden a no identificarse como tal, evitando así el estigma social del que serán objeto.

La heterogeneidad y las deficiencias en los sistemas de acogida de este colectivo que presenta un grave riesgo de vulnerabilidad y exclusión son un denominador común a nivel internacional. Son especialmente insuficientes los sistemas de atención a los/as solicitantes de protección internacional y a las personas refugiadas con diversidad funcional, ya sea de origen o causada durante el proceso migratorio y/o la huida. Son mínimos los programas no se identifica adecuadamente a solicitantes de asilo y personas refugiadas con discapacidad, deficiencias en los registros y en la formación y preparación de las y los profesionales que intervienen. A pesar de ello, las políticas locales son más sensibles y cercanas a las necesidades de estos colectivos que las políticas centrales.

La situación de las mujeres resulta más desconocida, oculta y vulnerable. En algunos casos se muestra la discapacidad como consecuencia del deterioro en la salud de las mujeres por una situación discriminatoria en el empleo precario que ocupan. La tendencia lleva a relacionar a las mujeres con discapacidad y a las mujeres migrantes con factores de vulnerabilidad y pobreza. $\mathrm{Y}$, sin embargo, estas mujeres se empoderan cuando tienen que apoyar a un hijo con discapacidad, la mujer con discapacidad migrante se hace activista, aprende a reivindicar sus derechos, desarrolla redes y genera importantes herramientas de inclusión.

Finalmente hay que indicar que desde planteamientos feministas se va impulsando el abordaje de este desde la necesidad de la perspectiva de la interseccionalidad, enfocando discapacidad, migración, clase social y género.

\section{REFERENCIAS BIBIBLIOGRÁFICAS}

Abdullahi, I. Wong, K. Mutch, R. et ál. (2019). Risk of Developmental Disorders in Children of Immigrant Mothers: A Population-Based Data Linkage Evaluation en Journal of pediatrics, 204, 275-284. DOI: 10.1016/j.jpeds.2018.08.047 
Ambrosini, M. (2013). "We are against a multi-ethnic society": policies of exclusion at the urban level in Italy. Ethnic and Racial Studies, 36 (1): 136-155.

Aumüller, J., Daphi, P., Biesenkamp, C. (2016). Die Aufnahme von Flüchtlingen in den Bundesländern und Kommunen. Behördliche Praxis und zivilgesell-schaftliches Engagement. Stuttgart: Robert Bosch Stiftung.

Ayre, K. Khalifeh, H. (2017). Immigrant women and women with learning disabilities have complex mental health needs and service use in the perinatal period. Evidence-Based Mental Health. 20, 5557.

Burns, N. (2017). The human right to health: exploring disability, migration and health, Disability \& Society, 32:10, 1463-1484

Calatayud, T. L. M., González, J. A. T., y Jiménez, L. O. (2017). Discapacidad y migración en el ámbito de las instituciones de educación superior: una lectura sobre los procesos de inclusión socio-educativa. Revista de Educación Inclusiva, 9(1), 1889-4208.

CAUCES (Cuadernos del Consejo Económico y Social) (2017). $N^{\circ} 34$. https://cendocps.carm.es/documentacion/2017_Cauces_N34.pdf 67-68.

Codina, L. (2018). Revisiones bibliográficas sistematizadas: Procedimientos gene- rales y Framework para Ciencias Humanas y Sociales. Máster Universitario en Comunicación Social. Barcelona: Departamento de Comunicación. Universitat Pompeu Fabra.

Comisión Europea (2016b). Die Städteagenda für die EU: Die europäischen Städte erhalten Mitspracherecht bei der Politikgestaltung der EU. Pressemit-teilung vom 30.5.2016. http://europa.eu/rapid/ press-release_IP-16-1924_de.htm.

Comisión Europea (2016c). Working Programme of the Urban Agenda for the EU http://ec.europa.eu/regional_policy/sources/policy/themes/urban-development/ agenda/urban-agenda-working-programme.pdf.

Díaz, E. y Huete, A.(2008). Las personas inmigrantes con discapacidad en España. Documentos del Observatorio Permanente de la Inmigración; 20. Ministerio de Trabajo e Inmigración. Madrid. Disponible en http://extranjeros.mitramiss. gob.es/es/ObservatorioPermanentelnmigracion/Publicaciones/fichas/archivos/ Las_personas_inmigrantes_con_discapacidad_en_Espaxa.pdf

Díaz, E. D., Huete A. y Jiménez, A.(2009). Inmigrantes con discapacidad en España. Zerbitzuan: Gizarte zerbitzuetarako aldizkaria = Revista de servicios sociales, (45), 135-150. 
Dossa, P. (2005). Racialized bodies, disabling worlds "they [service providers] always saw me as a client, not as a worker." Social Science \& Medicine, 60(11), 2527-2536. https://doi.org/10.1016/j.socscimed.2004.11.011

Dossa, P. (2009). Racialized bodies, disabling worlds: Storied lives of immigrant Muslim women. Toronto: University of Toronto Press.

Ferri, B.A. y Connor, D.J. (2005b). Tools of exclusion: Race, disability, and (re)segregated education. Teachers College Record, 107(3): 453-74.

Findley, S.E. (1988). The Directionality and Age Selectivity of the Health-Migration Relation: Evidence from Sequences of Disability and Mobility in the United States, International Migration Review, 22 (3), 4-29.

Gabel, S. L., S. Curcic, J. Powell, K. Khader, and L. Albee. (2009).. Migration and Ethnic Disproportionality in Special Education: An Exploratory Study. Disability \& Society 24 (5): 625-639. DOI: 10.1080/09687590903011063

Garcia, M. A., Downer, B., Crowe, M., y Markides, K. S. (2017). Aging and Disability Among Hispanics in the United States: Current Knowledge and Future Directions. Innovation in Aging, 1(2), igx020.

García, M. A. y Reyes A.M. (2018). Physical
Functioning and Disability Trajectories by Age of Migration Among Mexican Elders in the United States. Jornals of gerontology: Social Sciences, vol $73\left(n^{\circ} 7\right)$, 1292-1302. DOI:10.1093/geronb/gbw167

Garcia, M. A., Reyes, A. M., y Rote, S. (2019). Disability and the Immigrant Health $\mathrm{Pa}$ radox: Gender and Timing of Migration. In Contextualizing Health and Aging in the Americas, 249-269. DOl:https:/ /doi. org/10.1007/978-3-030-00584-9_12 (First Online 23 September 2018)

Gebhardt, D. (2015). Cities and immigrant citizenship. A comparison of local citi-zenship policies in Barcelona and $\mathrm{Mu}$ nich. GRITIM Working Paper Series. Nr. 26, Winter 2015. Barcelona: GRITIM.

Graefe, D. R; De Jong, G.F; y May, D.C.(2006). Work disability and migration in the early years of welfare reform. Population Research and Policy Review, 25 (4), 353-368. doi: 10.1007/s11113-006-9001-x

Handicap International and Help Age (2014). Hidden Victims of the Syrian Crisis: disabled, injured and older refugees, http:// www.handicap-international.us/hidden_ victims_of_syrian_crisis.

Hyangsook, C. (2017). A Case Study on Adaptation of Married Immigrant Women and Experience of Parenting of Children with Intellectual Disabilities. Journal of Inte- 
Ilectual Disabilities .19(4), 151-181

Human Rights Watch (2017). https://www. hrw.org/news/2017/01/18/greece-refugees-disabilities-overlooked-unders.

Khanlou, N; Mustafa, N; Vázquez, L.M; Davidson, D; y Yoshida, K. (2017). Mothering children with developmental disabilities: A critical perspective on health promotion, Health Care for Women International, 38:6, 613-634, DOI: 10.1080/07399332.2017.1296841

Leerkens, A. (2016). Back to the Poorhouse? Social protection and social control of unauthorised immigrants in the shadow of the welfare state. Journal of European Social Policy, 26 (2): 140-154.

McAuliffe, M. y Ruhs, M. (ed) (2018). Informe sobre las migraciones en el mundo 2018. Organización Internacional de las Migraciones. Ginebra: Organización Internacional de las Migraciones (OIM).

Mirza, M. (2011). Disablity and cross-border mobility: comparing resettlement experiences of Cambodian and Somali refugees with disabilites, en Disability \& Society 26, S. 521-535.

Mirza, M. y Heinemann, A. (2012). Service needs and service gaps among refguees with disabilities resettled in the United States, en Disablity \& Rehabilitation, 34, S. 542-552.

Montesino-Parra, N. (2016). Migración como discapacidad social: Trabajo Social con migrantes en Suecia. Trabajo Social Global. Revista de Investigaciones en Intervención social, 6 (10), 27-49.

Morcillo-Martínez, J. M. (2012). Discapacidad intelectual y violencia de género en mujeres migrantes:" la multidiscriminación". Portularia: Revista de Trabajo Social, (12), 1-11. DOI: 10.5218/prts.2012.0040

Oliver, C. y Singal, N.(2017). Migration, disability and education: reflections from a special school in the east of England, British Journal of Sociology of Education, 38:8, 1217-1229, DOI: 10.1080/01425692.2016.1273757

Organización de Naciones Unidas (2006). Convención de sobre los Derechos de las Personas con Discapacidad. Recuperado de http://www.un.org/spanish/disabilities/default.asp?id=497

Portera, A. (2014). Migration, intellectual disabilities and intercultural education. A qualitative study of young migrants of Italian origin in Germany en Revista electrónica interuniversitaria de formación del profesorado,17(2), 59-73 DOI: 10.6018/ reifop.17.2.197581

Peñaherrera, M., y Cobos, F. (2009). Inmigración y discapacidad: una aproximación a las" otras" discapacidades. Portularia IX, ADDENDA, 41-46

Roberts, K. y Harris, J. (2002). Disabled peo- 
ple in refugee and asylum seeking communities (Social care, race and ethnicity series), Bristol: The Policy Press.

Sajquim de Torres, M. y Lusk, M. (2018). Factors promoting resilience among Mexican immigrant women in the United States: Applying a positive deviance approach [Factores que promueven la resiliencia entre las mujeres inmigrantes mexicanas en los Estados Unidos: Aplicación de un enfoque de desviación positiva]. Estudios Fronterizos, 19, e005. https:// doi.org/10.21670/ ref.1805005

Scholten, P. y Penninx, R. (2016). The Multilevel Governance of Migration and Integration. S. 91-108 in Garcés-Mascareñas, B. / Penninx, R., (Hrsg.), Integration Processes and Policies in Europe. Context, Levels and Actors. Cham: Springer.

Schwalgin, S. (2017). Leerstellen benennen, Perspektiven aufzeigen: Geflüchtete mit Behinderungen in Deutschland. Key Note im Rahmen der Netzwerkkonferenz "Migration und Behinderung", 21. Berlin: Kleisthaus

Sheftel, M. G. (2017). Prevalence of disability among Hispanic immigrant populations: New evidence from the American community survey. Population Review, 56(1).

Sheftel, M., y Heiland, F. W. (2018). Disability crossover: Is there a Hispanic immigrant health advantage that reverses from working to old age?. Demographic Research, 39, 209-250. DOI:10.4054/ DemRes.2018.39.7

Smith-khan, L; Crock, M; Saul, B; y Mccallum (2014). To 'Promote, Protect and Ensure': Overcoming Obstacles in Forced Migration, Journal of Refugee Studies, 28 (1), 38-68. DOI:10.1093/jrs/feu020

Vertovec, S. (2007). Super-Diversity and its implications. Ethnic and Racial Studies, 30 (6), 1024-1054. DOI:10.1080/014198701599465

Ward, K., Amas, N. y Lagnado, J. (2008). Supporting disabled refugees and asylum seekers: opportunities for new approaches. London: Metropolitan Support Trust.

Wehmeyer, M.L. y Schwartz, M. (2001). Disproportionate representation of males in special education services: Biology, behavior, or bias?. Education and Treatment of Children, 24(1): 28-45.

Wong, E. H. S. (2012). Not Welcome A Critical Analysis of Ableism in Canadian Immigration Policy from 1869 to 2011. Critical Disability Discourses/Discours critiques dans le champ du handicap, 4.

Zelani, K., Bucken-Knapp, G., Hinnfors, J. y Spehar, A. (2016). Urban Governance of CEE mobility in European urban regions. Imagination Project Report. Rotterdam: Imagination Project. 
PARA CITAR ESTE ARTICULO:

Díaz Jiménez, R. M., de la Fuente Robles, Y. M., Muñoz Moreno, R. (2019). Migraciones y diversidad funcional. La realidad invisible de las mujeres. Collectivus, Revista de Ciencias Sociales, 6(1), 61-80. 
VOL. 6, NÚM. 1. ENERO-JUNIO (2019) 